\title{
Multiple appearing and vanishing aneurysms: primary angiitis of the central nervous system
}

\section{Case report}

\section{Misao Nishikawa, M.D., Hiroaki Sakamoto, M.D., Junsuke Katsuyama, M.D., Akira Hakuba, M.D., and Shuro Nishimura, M.D.}

Department of Neurosurgery, Osaka City General Hospital, Osaka, Japan; and Department of Neurosurgery, Osaka City University Medical School, Osaka, Japan

The authors present the case of a patient with ischemic episodes and recurrent intracerebral hemorrhages probably caused by primary angiitis of the central nervous system (CNS). An initial angiogram revealed multiple cerebral artery aneurysms as well as vascular wall irregularity; a second angiogram obtained 2 years later, however, did not demonstrate the previous aneurysms but instead showed new ones together with stenosis. Based on the histopathological findings and clinical course in this case, the multiple aneurysms appear to have been induced by vascular wall fragility and subsequent self-repair resulting from primary angiitis of the CNS. The authors present the histological and clinical characteristics of this unusual case of granulomatous, necrotizing CNS vasculitis.

Key Words * angiitis * intracerebral hemorrhage * multiple aneurysms * subarachnoid hemorrhage * central nervous system * children

The introduction of advanced imaging modalities has led to the establishment of clear diagnostic criteria and an improved prognosis for patients with primary angiitis of the central nervous system (CNS).[5] Nevertheless, the clinical manifestations of this disease can easily be confused with those of other conditions and diagnosis is still sometimes made only postmortem. We examined a young patient with a history of four ischemic episodes caused by intracerebral hemorrhages within a 5-year period. An initial angiogram demonstrated multiple cerebral artery aneurysms and vascular wall irregularity, but these aneurysms were not visible on a second angiogram, which instead showed new aneurysms as well as stenosis. The histopathological findings and clinical course of the disease strongly indicated a diagnosis of primary angiitis of the CNS.

\section{CASE REPORT}

History. This 10-year-old boy presented at our hospital with headache and left-sided hemiparesis. There were no abnormal findings on physical and neurological examinations but computerized tomography (CT) scanning revealed two lacunar infarctions in the right basal ganglia and a small area of low density next to the right lateral ventricle. Cerebral angiography showed eight fusiform or saccular-type aneurysms in the distal portion of the anterior cerebral artery (ACA), middle cerebral artery (MCA), and 
posterior cerebral artery along with vascular wall irregularities in several locations, suggesting preaneurysmal vasculitis (Fig. 1). The patient's blood cell count and chemistry findings were normal, as were the results of cerebrospinal fluid (CSF) analysis; immunological findings (antinuclear antigens, immunoglobulin, and component factors) in both blood and CSF were also normal. There was no evidence of collagen disease. In view of the transitory nature of the patient's symptoms, his young age, the risks associated with surgery, and the fact that we could not reach a definite diagnosis of primary CNS angiitis, we decided not to initiate aggressive treatment (despite his episodes of hemorrhagic and ischemic stroke), opting instead for close monitoring of his clinical course. The patient's headache and left-sided hemiparesis recurred every 2 or 3 months and although he underwent a complete examination at least once per year, the results did not vary from those obtained in the first examination.

Fig. 1. Left: Right common carotid artery (CCA) angiogram showing one fusiform and one saccular-type aneurysm in the distal portion of the ACA and MCA (arrows) as well as vascular wall irregularities (arrowheads). Center: Left CCA angiogram showing two fusiform aneurysms in the distal portion of the ACA and MCA (arrows) as well as vascular wall irregularity (arrowhead). Right: Left vertebral artery angiogram showing two fusiform and two saccular-type aneurysms in the distal portion of the posterior cerebral artery (arrows) as well as vascular wall irregularity (arrowhead).

First Operation. At the age of 12, the patient suddenly became comatose. Computerized tomography scanning showed an intracerebral hematoma in the frontal lobe. An angiogram of the left carotid artery demonstrated multiple aneurysms in the ACA and MCA, as had been the case with the previous angiogram; this time, however, two new aneurysms were also demonstrated in the MCA (Fig. 2 upper left). Surgery was performed to remove the hematoma and to coat the three aneurysms in the ACA and MCA, but the bleeding point in the aneurysm at the branch of the left callosomarginal artery could not be confirmed. The other aneurysm, located in the distal portion of the MCA, was not surgically treated. Postoperatively the patient was placed on a course of betamethasone (maximum initial dosage 16 $\mathrm{mg} /$ day, followed by tapering off) for 1 week to reduce the brain edema that ensued after surgery. After 1 month he was discharged from the hospital with normal neurological function. 

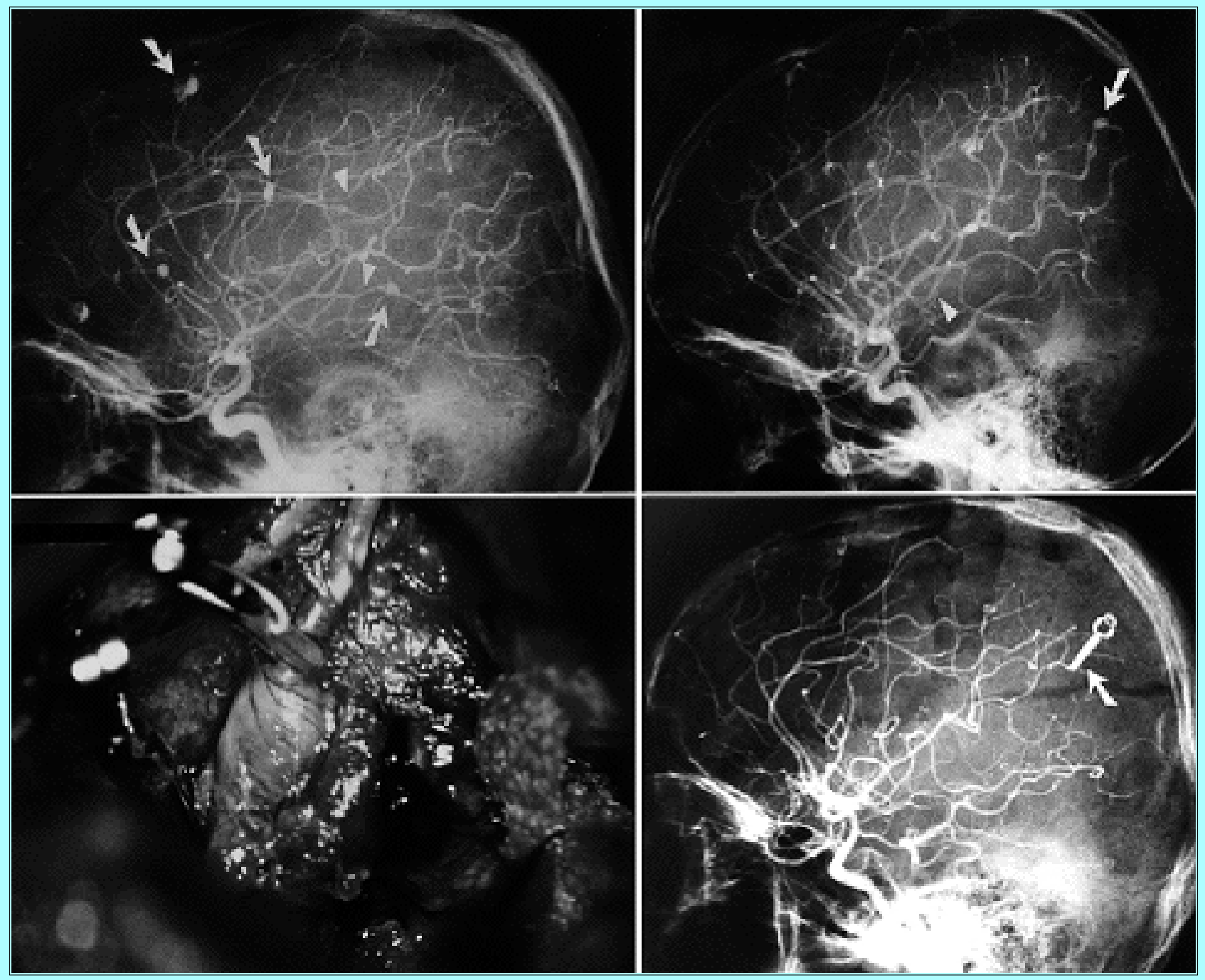

Fig. 2. Upper Left: Left CCA angiogram showing multiple aneurysms in the distal portion of the ACA and MCA (as in Fig. 1) together with two new aneurysms in the distal portion of the MCA (arrows). Arterial wall irregularity is also visible (arrowhead). Upper Right: Left CCA angiogram in which the three previously coated aneurysms and the untreated aneurysm are not seen, but instead demonstrating a new region of arterial wall irregularity (arrowhead) and a newly developed aneurysm (arrow). Lower Left: Intraoperative photograph demonstrating saccular aneurysms in the distal portion of the MCA. Lower Right: Left CCA angiogram showing successful clipping of the saccular aneurysm; the aneurysm is no longer demonstrated (arrow). No other aneurysms are discernible.

Second Operation. Six months after the patient was discharged, a follow-up angiogram demonstrated a newly developed aneurysm in the distal portion of the MCA, but it did not show the three previously coated aneurysms at the branch of the left callosomarginal artery or the two other aneurysms in the MCA (Fig. 2 upper right). Surgery was performed to clip one of the new MCA aneurysms (Fig. 2 lower left).

At the age of 13 years the patient underwent repeated angiography, which showed no opacification of the previously clipped aneurysm. This angiogram also showed irregularity of the left pericallosal artery wall (Fig. 2 lower right).

Third Operation. At the age of 15 the patient again became comatose, this time because of an extremely large hematoma $(5 \times 5 \mathrm{~cm})$ that had developed in his left frontal lobe. We evacuated the hematoma and removed the ruptured aneurysm by trapping the left pericallosal artery. Histopathological findings 
demonstrated some inflammatory cells infiltrating the wall of the aneurysm. We could not confirm the diagnosis based on these regional findings alone. The patient was in a vegetative state postoperatively and died of pneumonia approximately 2 years later at the age of 17 .

Autopsy Findings. The autopsy findings were multiple saccular and fusiform aneurysms as well as wall irregularities in the cerebral and spinal radicular arteries. Histological examination revealed no specific vascular abnormalities in the kidney, liver, or spleen, and there were no findings suggestive of angiitis in any other organ except the brain and spinal cord. Histological examination of the aneurysm walls showed infiltration by lymphocytes and macrophages along with chronic inflammatory reaction (Fig. 3 upper), but no signs of acute inflammation, mycotic embolization, or destruction of the elastic layer. Special staining of the vessel walls revealed no acid-fast bacilli or signs of viral infection. The vascular wall irregularities displayed numerous lymphocytes, granulomatous changes, and stenosis of the vascular lumen (Fig. 3 lower).

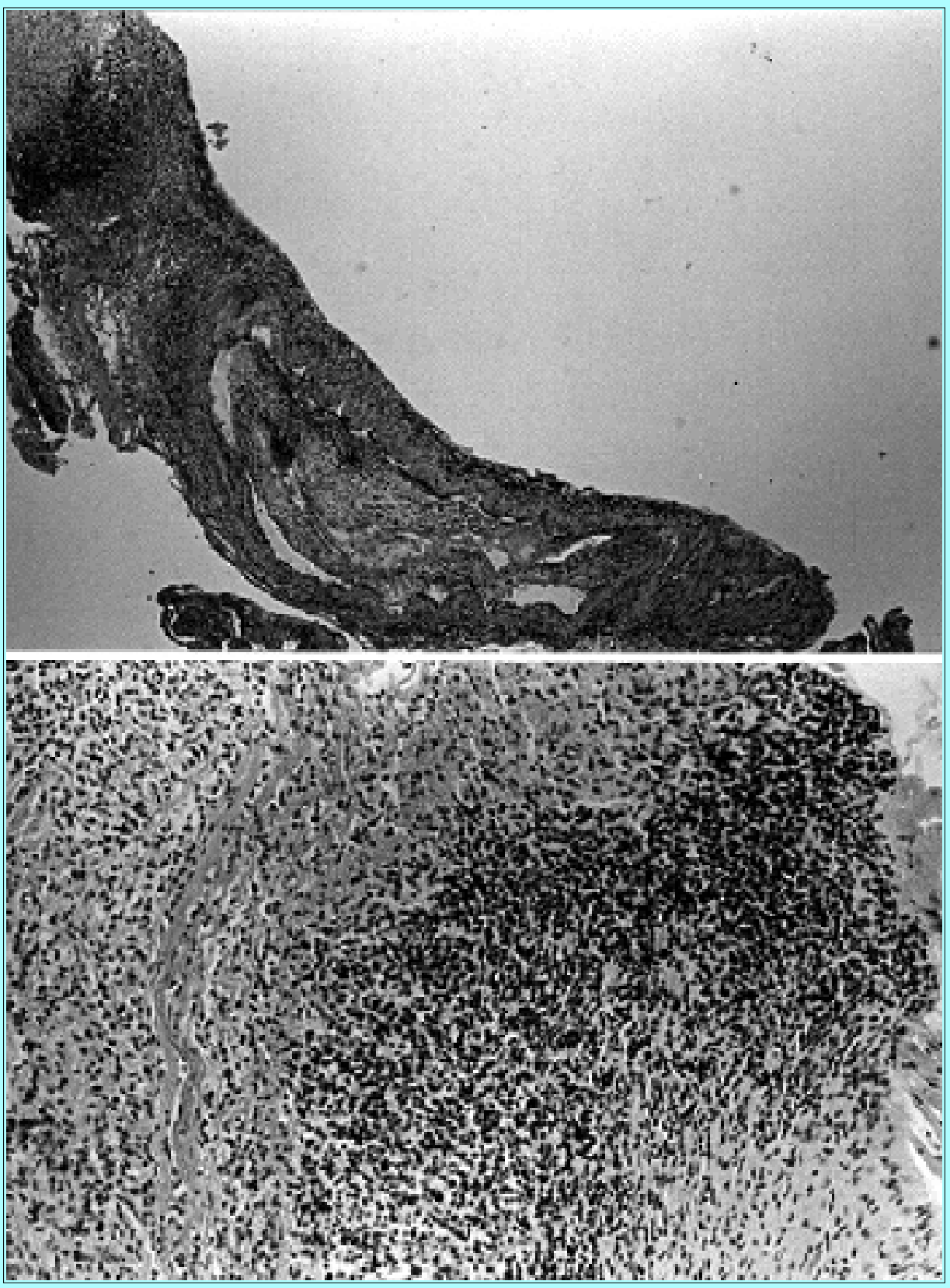

Fig. 3. Upper: Photomicrograph of the aneurysm wall showing infiltration by lymphocytes and macrophages together with chronic inflammatory reaction but no signs of acute 
inflammation or mycotic embolization. $\mathrm{H} \& \mathrm{E}$, original magnification X 40. Lower:

Photomicrograph of the wall of the vascular stenotic region showing numerous lymphocytes and granulomatous changes. The same findings were observed in the walls of the spinal cord aneurysms. H \& E, original magnification X 400.

\section{DISCUSSION}

\section{Clinical Manifestations and Diagnosis of Primary CNS Angiitis}

Although our patient's cerebral aneurysms were aggressively treated with surgical intervention, there were no definitive indications pointing to a diagnosis of primary angiitis of the CNS until the third operation, at which time histological inspection revealed many lymphocytes and granulomatous changes in the aneurysm walls. The clinical course of the patient's condition (ischemic episodes and recurrent intracerebral hemorrhages), the angiographic findings (wall irregularities and stenotic lesions), and the autopsy results (no abnormalities detected on CSF or laboratory testing) documented the presence and effects of angiitis that was largely confined to the CNS and characterized by vascular infiltration of epitheloid cells, giant cells, and lymphocytes in the absence of a demonstrable infectious agent.

A previous review of reported cases of primary CNS angiitis indicated that the average age of onset is between 15 and 96 years (mean 50.5 years), with a higher incidence in males (males 66\%:females 34\%). The cerebrum is the most frequently involved site (95\% of cases), followed by the pons or medulla oblongata (32\%), the cerebellum (18\%), and the spinal cord (16\%).[3,7] Headache is the most commonly reported symptom, but a wide variety of neurological signs and symptoms has also been described. Although a combination of focal and diffuse neurological deficits is characteristic of the disease, a few cases with purely focal presentation have also been reported.[3]

Diagnosis of primary angiitis of the CNS confronts the clinician with a problem of enormous complexity because of the wide variety of clinical presentations. Nonspecific signs of inflammation such as increased white blood cell count, increased erythrocyte sedimentation rate, and positive $\mathrm{C}$ reactive protein are occasionally observed but are not characteristic of the disease. Analysis of the CSF sometimes indicates increased protein concentration and mononuclear cells. Increased CSF pressure is a relatively common sign. Diagnosis is only possible after careful exclusion of other angiitides with CNS manifestation; subacute chronic meningoencephalitides; and neoplastic processes. Before reaching a definite diagnosis of primary CNS angiitis, we propose that the following criteria should be met: 1) the patient presents clinically with headaches and multifocal neurological deficits of at least 6 months' duration except in cases of devastating onset; 2) several areas of segmental arterial narrowing are demonstrated on cerebral angiography; 3) systemic inflammation or infection has been ruled out; and 4) a leptomeningeal/parenchymal biopsy specimen demonstrates vascular inflammation but no signs of infection, atherosclerosis, or neoplastic disease.[9]

The most common clinical manifestations of primary CNS angiitis are tissue ischemia produced by occlusion of the vascular lumen or by thrombus formation, and focal or multifocal cerebral infarction with or without hemorrhagic changes.[2] Intracerebral hemorrhages are caused by focal necrosis of the parenchymal artery wall. Subarachnoid hemorrhages can develop as a result of damage to the leptomeningeal arteries.[11]

In the case reported here, the patient's ischemic episodes indicated primary angiitis of the CNS; his recurrent intracerebral hemorrhages, caused by rupturing of appearing and disappearing aneurysms, are 
not atypical of the disease. The irregularities in the vascular wall appear to have been caused by chronic inflammation, which is indicative of vasculitis and preaneurysmal dilation. This inflammation also appears to have caused the weakening of the patient's arterial walls, which in turn seems to have led to his multiple aneurysms. Reported cases in which angiographic examination revealed the presence of clusters of saccular aneurysms[8] or in which multiple aneurysms were found to be associated with lymphomatoid granulomatosis[7,11] indicate that necrotic, angiodestructive infiltration by premalignant or malignant lymphoid cells can cause arterial walls to collapse from weakness.[12,13] Primary angiitis usually presents with ischemic lesions or hemorrhage caused by stenosis and vascular wall fragility. Hemorrhage of ruptured aneurysms resulting from angiitis has been reported in only three previous cases. $[8,11,14]$

To the best of our knowledge, ours is the first reported case of a ruptured aneurysm in the supratentorial region caused by primary angiitis of the CNS with autopsy-confirmed granulomatous changes in the aneurysm wall. It is possible that the aneurysm in our patient was not demonstrated on the subsequent angiogram because of self-repair of the vascular wall stimulated by postoperative infusion of steroid medication.

\section{Histopathological Features}

Granulomatous angiitis of the CNS is an uncommon form of vasculitis, first described as a distinct disease by Cravioto and Feigin[5] in 1959. There are only a few reports in the literature of primary CNS angiitis inducing subarachnoid or intracerebral hemorrhages.[1,4,6,14] Granulomatous angiitis associated with intracerebral hemorrhage and parenchymal edema was first described by Ozawa, et al.,[10] in 1995. This unique necrotizing vasculitis has been recognized as a distinct disease variously defined as "granulomatous angiitis of the central nervous system," "isolated angiitis of the central nervous system," or "primary angiitis of the central nervous system."[1,6,9,11-13]

In primary CNS angiitis, granulomatous changes--variable inflammatory infiltrates such as acute polynuclear leukocytes, chronic polynuclear lymphocytes, and multinucleated giant cells with focal fibrinoid necrosis--are often seen in the walls of leptomeningeal and parenchymal arteries and veins. This disease has well-defined characteristics that distinguish it as a clinicopathological entity.[15] However, its essential individuality is based on its difference from the other vasculitides that affect the CNS, notably collagen disease, sarcoidosis, giant cell (temporal) arteritis, granulomatous angiitis with Hodgkin's disease, allergic angiitis, infectious angiitis, Takayasu's disease, and rheumatoid arteritis. In the case reported here, the histopathological findings in the aneurysms walls in the cerebrum and spinal cord indicated infiltration by chronic polynuclear lymphocytes and multinucleated giant cells; no vascular abnormalities were found in any other organs. It was these findings that confirmed the diagnosis of primary angiitis of the CNS.

The cause of primary CNS angiitis remains unknown. The presence of granuloma and the absence of antibodies and immune complexes in the vessel walls suggest a cell-mediated disorder of the immune system. The reason behind the activation of cell-mediated inflammation is unclear. There are no reports of clinical, serological, or hereditary features that would indicate an underlying autoimmune process. However, reductions in serum complement levels and the effectiveness of steroid medication therapy reported by previous authors suggest that the autoimmune process may play a causal role in this disease.[7] 
Because the cause of primary CNS angiitis remains unknown, therapy is directed at the inflammatory process. Steroid medications are therefore the primary form of medical management. In progressive or resistant cases, cytotoxic agents such as azathioprine or more recently cyclophosphamide have been used.[6] Recognition of this therapeutic possibility is important, especially because immunosuppressant drugs may halt the underlying process and prevent the potentially irreversible consequences of neural damage associated with this disease. We were unable to provide our patient with the most appropriate treatment because we did not fully grasp the underlying pathophysiological mechanisms of his angiitis, which had the perplexing property of resulting in both hemorrhagic and ischemic strokes.

Although we are unable to offer a definitive explanation for the vanishing aneurysm in our patient, we speculate that the wall of this aneurysm may have been repaired when the patient's immune system was suppressed by administration of steroid medication (betamethasone). Trapping and resection appear advisable in treating hemorrhages caused by rupturing of angiitis-related aneurysms because the latter are in fact pseudoaneurysms that tend to occur when inflammatory changes in vascular walls lead to structural fragility.

\section{References}

1. Biller J, Loftus CM, Moore SA, et al: Isolated central nervous system angiitis first presenting as spontaneous intracranial hemorrhage. Neurosurgery 20:310-315, 1987

2. Burger PC, Burch JG, Vogel FS: Granulomatous angiitis: an unusual etiology of stroke. Stroke 8:29-35, 1977

3. Calabrese LH, Mallek JA: Primary angiitis of the central nervous system: report of 8 new cases, review of the literature, and proposal for diagnostic criteria. Medicine 67:20-39, 1988

4. Clifford-Jones RE, Love S, Gurusinghe N: Granulomatous angiitis of the central nervous system: a case with recurrent intracerebral haemorrhage. J Neurol Neurosurg Psychiatry 48:1054-1056, 1985

5. Cravioto H, Feigin I: Noninfectious granulomatous angiitis with a predilection for the nervous system. Neurology 9:559-609, 1959

6. Cupps TR, Moore PM, Fauci AS: Isolated angiitis of the central nervous system: prospective diagnostic and therapeutic experience. Am J Med 74:97-105, 1983

7. Fukasawa I, Mitsuka S, Nukui H, et al: Granulomatous angiitis of the central nervous system--case report. Neurol Med Chir 33:386-390, 1993

8. Griffin J, Price DL, Davis L, et al: Granulomatous angiitis of the central nervous system with aneurysms on multiple cerebral arteries. Trans Am Neurol Assoc 98:145-148, 1973

9. Moore PM: Diagnosis and management of isolated angiitis of the central nervous system. Neurology 39:167-173, 1989

10. Ozawa T, Sasaki O, Sorimachi T, et al: Primary angiitis of the central nervous system: report of two cases and review of the literature. Neurosurgery 36:173-179, 1995

11. Sandhu R, Alexander S, Hornabrook RW, et al: Granulomatous angiitis of the CNS. Arch Neurol 
36:433-435, 1979

12. Scully RE, Galdabini JJ, McNeely BU: Case records of the Massachusetts General Hospital. Case 43-1976. N Engl J Med 295:944-950, 1976

13. Scully RE, Mark EJ, McNeely BU: Case record of the Massachusetts General Hospital. Case 35-1985. N Engl J Med 313:566-575, 1985

14. Yoong MF, Blumbergs PC, North JB: Primary (granulomatous) angiitis of the central nervous system with multiple aneurysms of spinal arteries. Case report. J Neurosurg 79:603-607, 1993

15. Zimmerman RS, Young HF, Hadfield MG: Granulomatous angiitis of the nervous system: a case report of long-term survival. Surg Neurol 33:206-212, 1990

Manuscript received November 26, 1996.

Accepted in final form August 18, 1997.

Address reprint requests to: Misao Nishikawa, M.D., and Hiroaki Sakamoto, M.D., Department of Neurosurgery, Osaka City General Hospital, 2-13-22 Miyakojima-hondori, Miyakojima-ku, Osaka 534, Japan. 